\title{
GEARBOX VIBRATORY ANALYSIS USING CARRYING, COUPLING AND SLAVE SUBSTRUCTURES
}

\author{
Abbes, M. S.; Fakhfakh, T. \& Haddar, M. \\ Mechanics Modelling and Production Research Unit, Mechanical Engineering Department \\ National School of Engineers of Sfax (ENIS), BP. W. 3038, Sfax, Tunisia \\ E-mail: $\underline{m \text { haddar@voila.fr }}$
}

\begin{abstract}
Vibration analysis of complex structures is usually accomplished by analysing its finite element model. When the total number of degrees of freedom in the model is too large, we deal with large-scale eigenvalue problems. The classical approach for the solution of such problems consists in reducing the number of unknowns, allowing reducing the computational cost of eigensolver. Component mode synthesis or dynamic substructuring methods are appropriate tools for this reduction. In this paper, a novel approach in dynamic substructuring for numerical simulation of complex structures is presented and three type of substructure are considered. (1) Carrying substructure (plates and beams), (2) coupling substructure (organs joining carrying substructures), (3) slave substructure (elements connected to the carrying substructure). This approach is based on the use of the Craig-Bampton decomposition of the admissible displacement field. A numerical example is presented. A gearbox with elastic casing, two transmission shafts and one helical gear pair is analyzed and concepts for reduced noise and vibration are identified.
\end{abstract}

Key Words: Substructuring Method, Gearbox Modelling, Numerical Simulation, Eigenvalues

\section{INTRODUCTION}

There are several methods in dynamic substructuring for numerical simulation of complex structures in the modal frequency range [1]. The Craig-Bampton method [2] is very efficient to calculate the dynamical response of a complex structure (modelled by finite element method). This method was initially developed for discretised systems. Hou [3] and Graig [4] presented summaries of various developments in component mode synthesis. The fixedinterface component mode synthesis developed by Hutry [5] and refined by Craig and Bampton [2] generally produces very accurate eigensolutions for a given number of degreesof-freedom (d.o.f), but requires that all of the original interface displacement co-ordinates must be retained in final assembled structure model.

Much effort has been expended during the design process of a gearbox structure to predict and reduce the noise radiated by its casing [6,7]. Previous studies have yielded a vast literature on this topic and in particular, a remarkable variety of mathematical models as discussed in reference [8]. Todd and Rajendra [9] have developed a new methodology based on a substructuring mobility approach in order to focus on the influence of bearing design on the dynamical behaviour of gearbox. A simplified gearbox structure is analytically and experimentally studied. Stiffness and mass gear casing matrices has been incorporate into an overall gearbox model by Adline [10] using fixed interface component mode.

In this paper, an efficient substructuring method based on the use of the Craig-Bampton decomposition is presented. In this method it is assumed that the studied structure is partitioned in $\mathrm{N}_{\mathrm{S}}$ substructures (carrying, coupling and slave). Each substructure is analysed independently and its eigenfrequencies and mode shapes are obtained. This procedure allows 
us to recover the whole vibratory characteristics of the complete structure. The proposed method is implemented on a helical gear transmission fitted with elastic casing. The eigenvalue problems have been computed and results are compared with direct finite element analysis of the full model. Eigensensitivity due to addition of stiffener on the casing elastic front plate is analysed.

\section{THE DYNAMIC SUBSTRUCTURING METHOD (DSM)}

A substructure is defined as a particular subportion of a vibrating assembly structure as shown in Fig. 1. Every carrying substructure occupies a bounded domain $\Omega^{i}$ with boundary defined as:

$$
\delta \Omega^{i}=\bigcup_{k} \Gamma_{f}^{i} \oplus \Gamma_{g}^{i} \oplus \Gamma_{c}^{i}
$$

where $\Gamma_{f}^{i}$ is the free part of the boundary and $\Gamma_{g}^{i}$ is the boundary with external body force field. $\Gamma_{c}^{i}$ represents the coupling interface boundary that separates two adjacent carrying substructures. Finite element meshes of $\Omega^{i}$ are considered and assumed to be compatible at coupling interface $\Gamma_{c}^{i}$. For a bounded domain $\Omega^{i}$, slave substructures are introduced as a concentrated mass $m^{j}$ and/or stiffener $\Gamma_{S}$ connected to the carrying substructure $S_{i}$ at any number of discrete points.

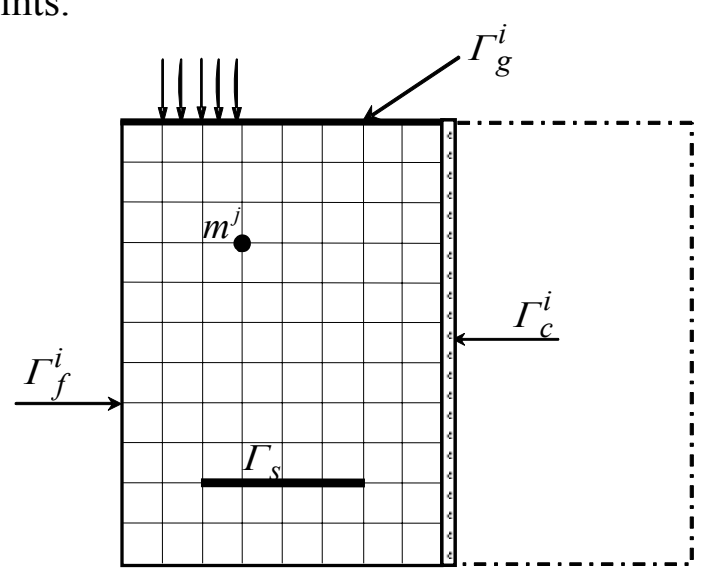

Figure 1: Structure decomposed into 3 substructure types $\left(S_{i}, \Gamma_{c}^{i}\right.$ and $\left.\left(\Gamma_{s}, m^{j}\right)\right)$.

Kinetic and strain energies of the substructures can be written as:

$$
\begin{aligned}
& T_{S}=\frac{1}{2}\{\dot{q}\}_{S}^{T}[M]_{S}\{\dot{q}\}_{S} \\
& U_{S}=\frac{1}{2}\{q\}_{S}^{T}[K]_{S}\{q\}_{S}
\end{aligned}
$$

where the subscript $S$ refers to the carrying, coupling and slave substructures. Mass and stiffness carrying substructure matrices are assembled with those of corresponding slave substructures. The modal reduction of each substructure enhanced with slave substructures is obtained by the projection of mass $[\tilde{M}]_{S}$ and rigidity $[\tilde{K}]_{S}$ matrices onto the Ritz basis: 


$$
\{q\}_{S}=\left[\begin{array}{l}
q_{B} \\
q_{I}
\end{array}\right]=\left[\begin{array}{cc}
I & 0 \\
\Psi_{C} & \Phi_{N}
\end{array}\right]\left[\begin{array}{l}
q_{B} \\
\xi_{N}
\end{array}\right]=\left[T_{F}\right]_{S}\{\zeta\}_{S}
$$

Here, suffix $B$ and $I$ respectively refer to the boundary and internal d.o.f. D.o.f $\xi_{N}$ are generalized coordinates related to the natural modes of the substructure. The columns of the matrix $\Phi_{N}$ are the natural modes of the substructure with fixed interface boundaries. They are obtained by solving the eigenproblem:

$$
\left[\tilde{K}_{I I}-\omega^{2} \tilde{M}_{I I}\right]\left\{\Phi_{I}\right\}=0
$$

where $K_{I I}, M_{I I}$ are the appropriate partitions of $[\tilde{K}]_{S}$ and $[\tilde{M}]_{S}$. The matrix $\Psi_{C}$ is a matrix of constraint modes of the substructure. Each column represents the values assumed by d.o.f at the internal nodes for a unit value of one of the degrees of freedom at an interface boundary node. These are given by the solution of

$$
\begin{gathered}
{\left[\tilde{K}_{I I}\right]\left\{q_{I}\right\}+\left[\tilde{K}_{I B}\right]\left\{q_{B}\right\}=0} \\
\left\{q_{I}\right\}=\left[\tilde{K}_{I I}\right]^{-1}\left[\tilde{K}_{I B}\right]=\left[\Psi_{C}\right]\left\{q_{B}\right\}
\end{gathered}
$$

The transformed mass and stiffness matrices used in the DSM are given by:

$$
\begin{gathered}
{[\overline{\tilde{M}}]_{S}=\left[T_{F}\right]_{S}^{T}[\tilde{M}]_{S}\left[T_{F}\right]_{S}=\left[\begin{array}{cc}
\overline{\tilde{M}}_{B B} & \overline{\tilde{M}}_{B N} \\
\overline{\tilde{M}}_{N B} & \overline{\tilde{M}}_{N N}
\end{array}\right]} \\
{[\overline{\tilde{K}}]_{S}=\left[T_{F}\right]_{S}^{T}[\tilde{K}]_{S}\left[T_{F}\right]_{S}=\left[\begin{array}{cc}
\overline{\tilde{K}}_{B B} & 0 \\
0 & \tilde{\tilde{K}}_{N N}
\end{array}\right]}
\end{gathered}
$$

with $\overline{\tilde{M}}_{N N}=\Phi_{N}^{T} \tilde{M}_{I I} \Phi_{N}$ and $\overline{\tilde{K}}_{N N}=\Phi_{N}^{T} \tilde{K}_{I I} \Phi_{N}$ are diagonal matrices. $\overline{\tilde{K}}_{B B}=\tilde{K}_{B B}+\tilde{K}_{B I} \Psi_{C}$, where $\tilde{K}_{B B}, \tilde{K}_{B I}$ are partitions of $[\tilde{K}]_{S}$ which is the stiffness matrix of the substructure in terms of the interface boundary degrees of freedom.

For two substructure systems (denoted 1 and 2) the total kinetic and strain energies are given below as:

$$
\begin{aligned}
& T=\frac{1}{2}\{\dot{\xi}\}^{T}[\overline{\tilde{M}}]\{\dot{\xi}\} \\
& U=\frac{1}{2}\{\xi\}^{T}[\overline{\tilde{K}}]\{\xi\}
\end{aligned}
$$

where

$$
[\overline{\tilde{M}}]=\left[\begin{array}{ccc}
\overline{\tilde{M}}_{B B}^{1}+\overline{\tilde{M}}_{B B}^{2} & \overline{\tilde{M}}_{B N}^{1} & \overline{\tilde{M}}_{B N}^{2} \\
\tilde{\tilde{M}}_{N B}^{1} & \overline{\tilde{M}}_{N N}^{1} & 0 \\
\overline{\tilde{M}}_{N B}^{2} & 0 & \overline{\tilde{M}}_{N N}^{2}
\end{array}\right] ;[\overline{\tilde{K}}]=\left[\begin{array}{ccc}
\overline{\tilde{K}}_{B B}^{1}+\overline{\tilde{K}}_{B B}^{2} & 0 & 0 \\
0 & \overline{\tilde{K}}_{N N}^{1} & 0 \\
0 & 0 & \overline{\tilde{K}}_{N N}^{2}
\end{array}\right] ;\{\xi\}=\left[q_{B} \xi_{N}^{1} \xi_{N}^{2}\right]
$$


The reduced stiffness $\overline{\tilde{K}}$ and mass $\overline{\tilde{M}}$ matrices are finally assembled with those of coupling substructures $\left(K_{c}\right.$ and $\left.M_{c}\right)$. Note that only interface boundary degrees of freedom are concerned with this assembly and the corresponding rigidity and mass matrices are given by:

$$
\begin{aligned}
\overline{\tilde{K}}_{B B} & =\sum_{n=1}^{2} \overline{\tilde{K}}_{B B}^{n}+K_{c} \\
\overline{\tilde{M}}_{B B} & =\sum_{n=1}^{2} \overline{\tilde{M}}_{B B}^{n}+M_{c}
\end{aligned}
$$

$n$ denotes the number of carrying substructures.

The above procedure can be generalized for $\mathrm{N}_{\mathrm{S}}$ substructures and the reduced eigenvalue problems of an assembled structure can be written as follows:

$$
\left([\overline{\tilde{K}}]-\omega^{2}[\overline{\tilde{M}}]\right)\{\xi\}=0
$$

The derived set of system natural frequencies and corresponding mode shapes can then be used to compute dynamic response of the assembled structure.

\section{NUMERICAL SIMULATION}

\subsection{Study of a gearbox}

The above-described method is implemented on a complex structure that consists on a single stage gearbox fitted with a helical gear as shown in Figure 2. The main characteristics of the gear pair are presented in Table I. The casing having parallelepiped form is made of five rigid panels (the frame), and one elastic panel on which the driving and driven shafts are mounted. The elastic panel is supposed to be clamped to the rigid panel at all boundaries. The two shafts connected to each other with a gear pair represent the first substructure, while the elastic front plate represents the second substructure.

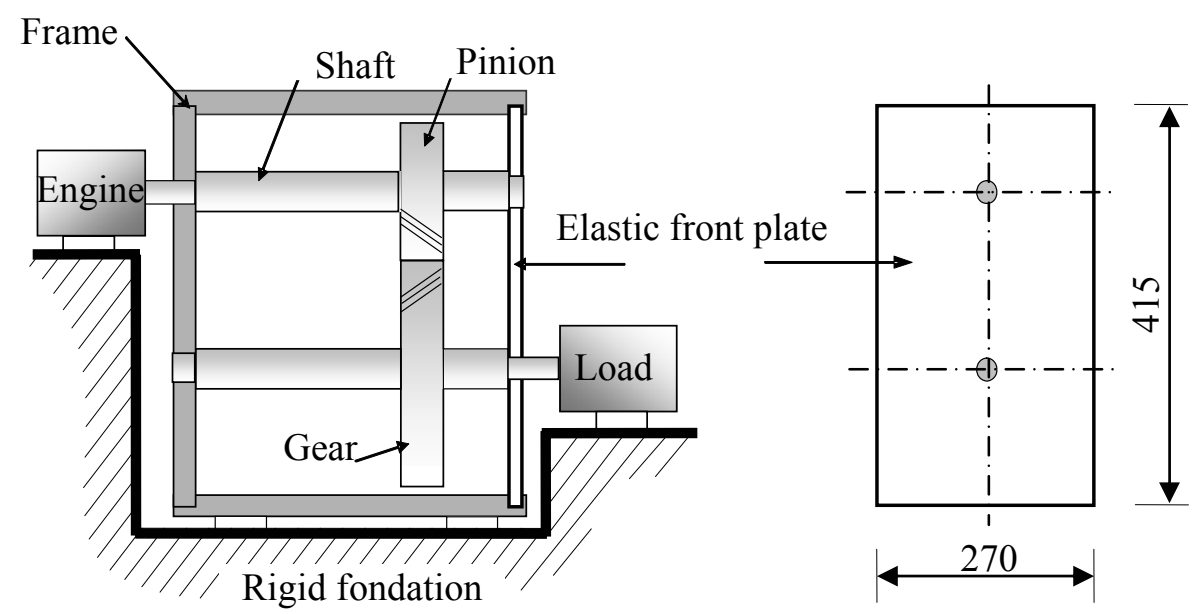

Figure 2: Schematic of a simplified gearbox. 
Table I: Gear pair data.

\begin{tabular}{|c|c|c|}
\hline & Pinion & Gear \\
\hline Base radii $R_{b}$ & $60.2 \mathrm{~mm}$ & $84.2 \mathrm{~mm}$ \\
\hline Normal module $\mathrm{m}_{\mathrm{n}}$ & \multicolumn{2}{|c|}{$3.5 \mathrm{~mm}$} \\
\hline Transverse pressure angle & \multicolumn{2}{|c|}{$23^{\circ}$} \\
\hline Face width & \multicolumn{2}{|c|}{$35 \mathrm{~mm}$} \\
\hline Base helix angle & \multicolumn{2}{|c|}{$19^{\circ}$} \\
\hline Centre distance & \multicolumn{2}{|c|}{$158 \mathrm{~mm}$} \\
\hline Average mesh stiffness & \multicolumn{2}{|c|}{$5 \cdot 10^{8} \mathrm{~N} / \mathrm{m}$} \\
\hline
\end{tabular}

The pinion and the gear are modelled with concentrated masses and rotational inertia. They are coupled by a $12 \times 12$ stiffness matrix defined from the geometrical characteristics of the gear pair and from the mean value $k_{m}$ of mesh stiffness $k(t),[11]$.

$$
\begin{gathered}
{\left[K_{g}\right]=k_{m}\{T\}^{T}\{T\}} \\
\{T\}=\left\{-\cos \beta_{b}, 0,-\sin \beta_{b},-R_{b 1} \sin \beta_{b},-R_{b 1} \operatorname{tg} \alpha \sin \beta_{b}, R_{b 1} \cos \beta_{b},\right. \\
\left.+\cos \beta_{b}, 0,+\sin \beta_{b},-R_{b 2} \sin \beta_{b},-R_{b 2} \operatorname{tg} \alpha \sin \beta_{b}, R_{b 2} \cos \beta_{b}\right\}
\end{gathered}
$$

where $R_{b 1}$ and $R_{b 2}$ are the base radii of the pinion and gear, $\alpha$ and $\beta_{b}$ denote respectively the pressure and the base helix angles. The periodic mesh stiffness $k(t)$ is obtained through measurement or calculation (e.g., sinusoidal or rectangular wave) [12].

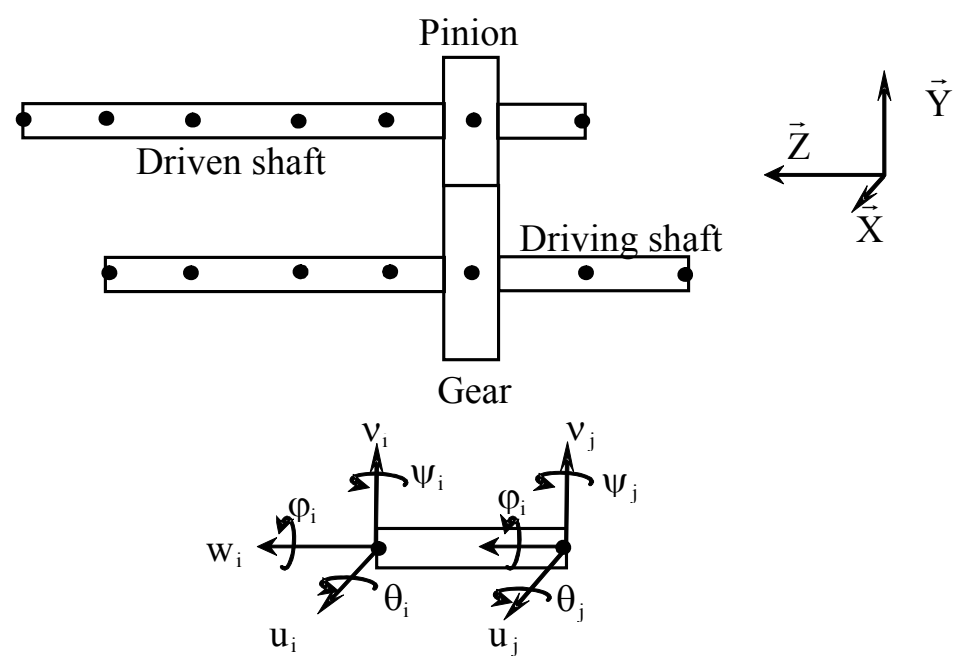

Figure 3: Finite element model of the first substructure (shafts and gear pair).

The shafts are discretised using beam finite elements with 2 nodes and 6 d.o.f. per node. The beam section is constant in $z$ direction. These elements include the effects of torsion, bending and traction-compression [13]. The generalised displacement of the $\mathrm{j}^{\text {th }}$ node is given by $^{T}\left\{q_{j}\right\}=\left\{u_{j}, v_{j}, w_{j}, \varphi_{j}, \psi_{j}, \theta_{j}\right\}$ where $\left(u_{j}, \psi_{j}\right)$ and $\left(v_{j}, \varphi_{j}\right)$ are respectively the beam bending in the $x-y$ plane and in the $y-z$ plane, $w_{j}$ and $\theta_{j}$ are respectively the d.o.f associated to the axial and torsional deformations, Fig. 3. 
The discretisation of the first substructure $[14,15]$ leads to the following movement matrix equation:

$$
\left[K_{r}\right]\left\{W_{r}\right\}+\left[M_{r}\right]\left\{\ddot{W}_{r}\right\}=\{0\}
$$

where $\left[K_{r}\right]$ and $\left[M_{r}\right]$ are respectively the rigidity and mass matrix of the transmission (shaft and pair of gear) and $\left\{W_{r}\right\}$ the nodal displacement vector.

The second substructure was discretised using plate finite element [14]. The discretised expression of the deformation and kinetic energy allows writing the following movement matrix equation:

$$
\left[K_{h}\right]\left\{W_{h}\right\}+\left[M_{h}\right]\left\{\ddot{W}_{h}\right\}=\{0\}
$$

$\left[K_{h}\right]$ and $\left[M_{h}\right]$ are respectively the stiffness and mass matrix of the casing.

$\left\{W_{h}\right\}$ and $\left\{\ddot{W}_{h}\right\}$ are nodal displacement and acceleration vectors.

\subsection{Eigenproblem computation}

From the recovered eigenvalues of each substructure, the generalised natural frequencies and mode shapes of the assembled gearbox are obtained.

Table II list the gearbox natural frequencies obtained with the proposed dynamic substructuring method DSM and the direct finite element analysis FEM. An excellent agreement is observed with error $\varepsilon$ in $\omega_{i}$ being less than $3 \%$ for the first 7 modes. However, this error can reach $8 \%$ at higher modes.

Some of the pertinent mode shapes of the assembled gearbox are shown in Fig. 4. It was found that the eigenmodes could be classified into three classes. In the first class, the dominant displacement is located in the shafts as presented in Figures 4-(a) and 4-(b). This displacement is influenced by the gearmesh element that connects the two shafts. In the second class only the elastic front plate is subject to an important bending deflection (Fig. 4-(c) and 4-(d)) whereas the shafts don't move. In the third class a combined modal deflection of both the shafts and the front plate is observed which confirms the interaction between these two substructures. Figures 4-(d) and 4-(e) show an example of this class.

Table II: Natural frequencies of simplified gearbox obtained from DSM and FEM.

\begin{tabular}{cccc}
\hline \multicolumn{4}{c}{ Gearbox frequency, $\left.\omega_{\mathbf{i}} \mathbf{( H z}\right)$} \\
\hline Mode $\boldsymbol{i}$ & DSM & FEM & $\varepsilon \mathbf{~}$ \\
\hline $\mathbf{1}$ & 286 & 288 & 0.7 \\
$\mathbf{2}$ & 638 & 650 & 1.8 \\
$\mathbf{3}$ & 935 & 945 & 1.3 \\
$\mathbf{4}$ & 950 & 960 & 1.0 \\
$\mathbf{5}$ & 1160 & 1185 & 2.1 \\
$\mathbf{6}$ & 1175 & 1200 & 2.0 \\
$\mathbf{7}$ & 1260 & 1285 & 1.9 \\
$\mathbf{8}$ & 1809 & 1890 & 4.2 \\
$\mathbf{9}$ & 1947 & 2060 & 5.4 \\
$\mathbf{1 0}$ & 1960 & 2105 & 6.8 \\
$\mathbf{1 1}$ & 2032 & 2180 & 6.7 \\
$\mathbf{1 2}$ & 2049 & 2195 & 6.6 \\
$\mathbf{1 3}$ & 2078 & 2240 & 7.2 \\
$\mathbf{1 4}$ & 2268 & 2450 & 7.4 \\
$\mathbf{1 5}$ & 2315 & 2490 & 7.0 \\
\hline
\end{tabular}




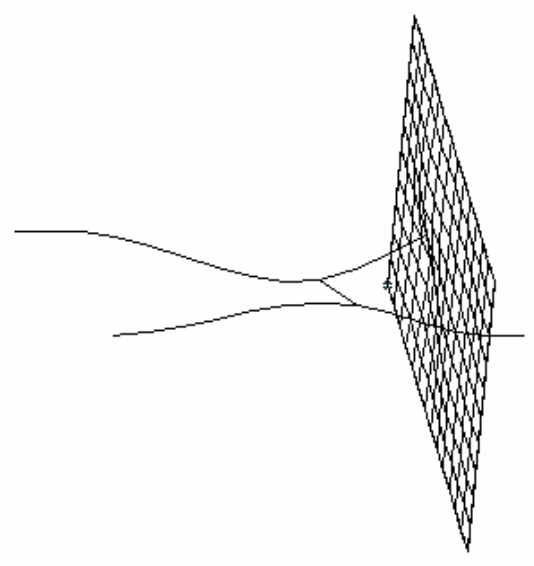

(a) Mode 2

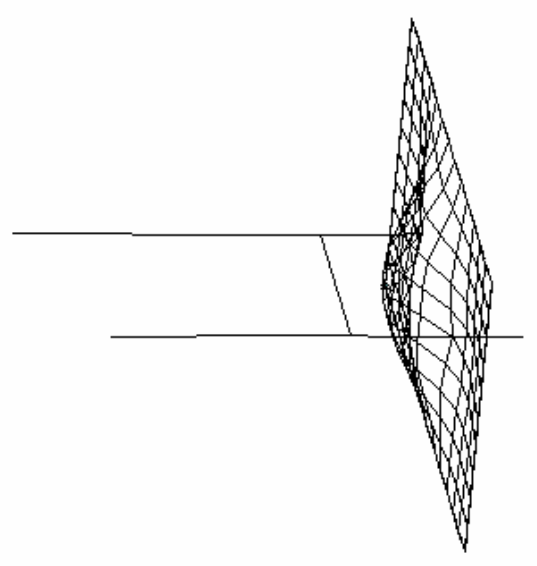

(c) Mode 7

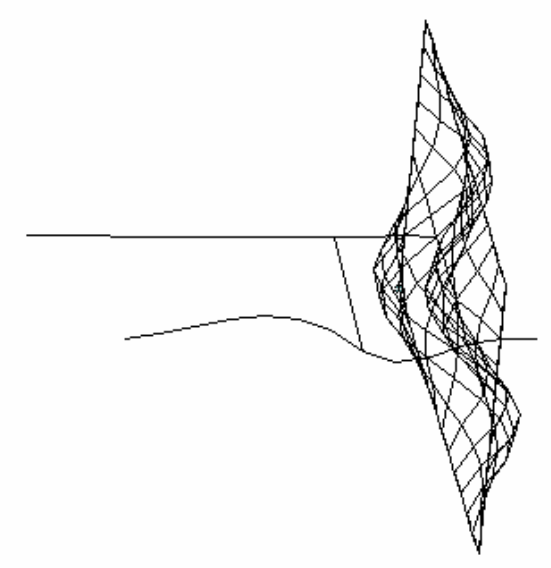

(e) Mode 11

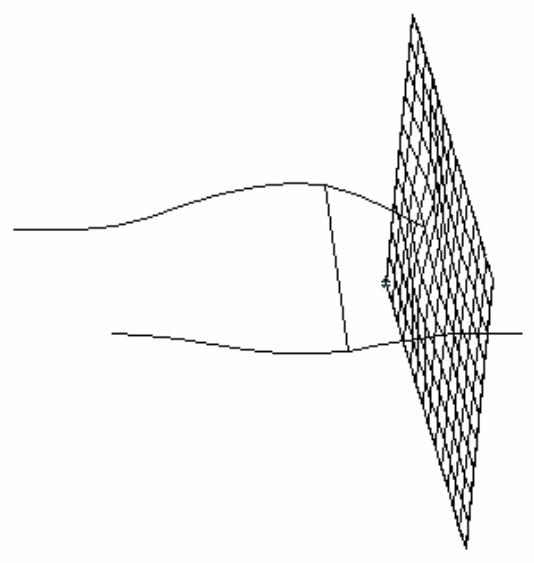

(b) Mode 6

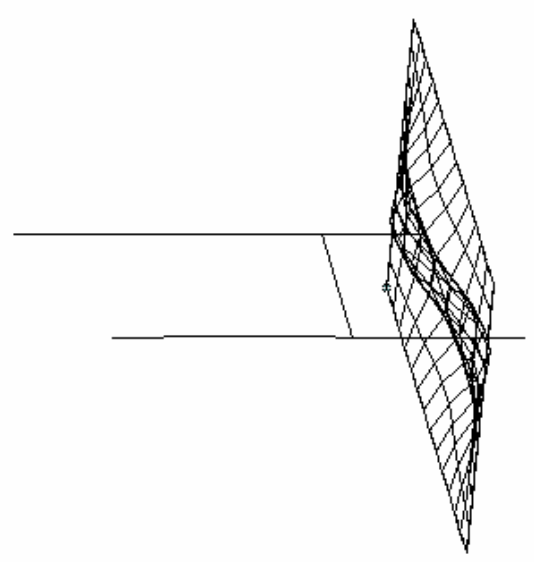

(d) Mode 9

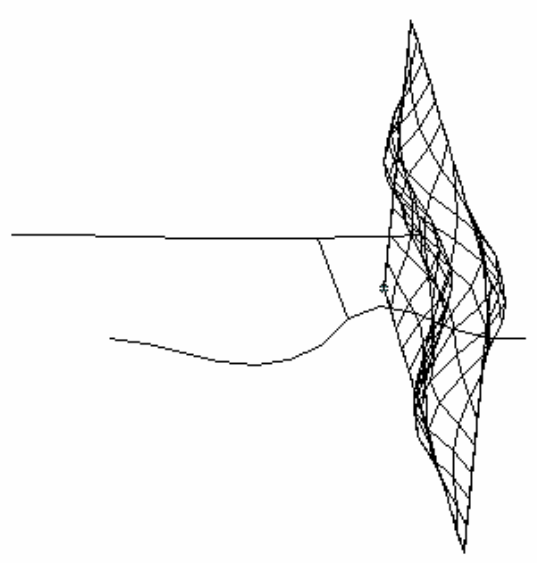

(f) Mode 12

Figure 4: Mode shapes of the simplified gearbox: the two shafts, gearmesh element and the elastic front plate. 


\subsection{Effect of stiffener on the casing vibratory behaviour}

The implementation of DSM allows us to look at a difference in vibratory responses between different design configurations made on one carrying substructure without redoing all the computation stage, as it must be done by the FEM. This particular specification is well adapted for vibro-acoustic parametric design studies.

In the case of the studied gearbox example, the front plate is fitted with steel stiffener considered as a slave substructure (Fig. 5) that is modelled by beam elements having 6 d.o.f. per node. Using the dynamic substructuring method, the first fifteen natural frequencies were determined. They are shown in Table III along with the previous values for the simplified gearbox without stiffener.

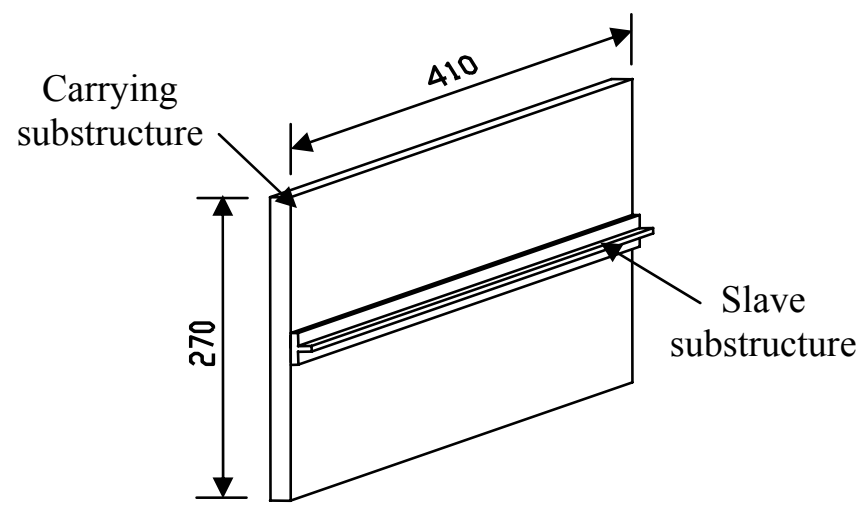

(a)

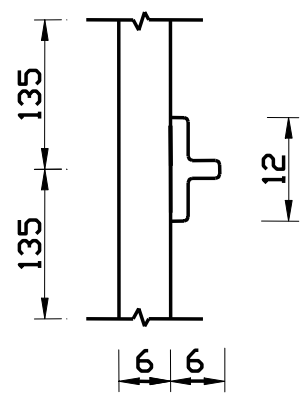

(b)

Figure 5: Stiffened elastic panel (dimensions are in $\mathrm{mm}$ ):

(a) front plate with stiffener, and (b) cross-section of stiffened front plate.

Table III: Natural frequencies of the simplified gearbox obtained from DSM.

\begin{tabular}{ccc}
\hline \multicolumn{3}{c}{ Gearbox frequency, $\omega_{\mathrm{i}} \mathbf{( H z )}$} \\
\hline \multirow{2}{*}{ Mode $\boldsymbol{i}$} & $\begin{array}{c}\text { Housing } \\
\text { without stiffener }\end{array}$ & $\begin{array}{c}\text { Housing } \\
\text { with stiffener }\end{array}$ \\
\hline $\mathbf{1}$ & 286 & 593 \\
$\mathbf{2}$ & 638 & 851 \\
$\mathbf{3}$ & 935 & 946 \\
$\mathbf{4}$ & 950 & 982 \\
$\mathbf{5}$ & 1160 & 1130 \\
$\mathbf{6}$ & 1175 & 1300 \\
$\mathbf{7}$ & 1260 & 1792 \\
$\mathbf{8}$ & 1809 & 1891 \\
$\mathbf{9}$ & 1960 & 2053 \\
$\mathbf{1 0}$ & 1947 & 2068 \\
$\mathbf{1 1}$ & 2032 & 2105 \\
$\mathbf{1 2}$ & 2049 & 2167 \\
$\mathbf{1 3}$ & 2078 & 2320 \\
$\mathbf{1 4}$ & 2268 & 2379 \\
$\mathbf{1 5}$ & 2315 & 2458 \\
\hline
\end{tabular}

It is noted that adding a stiffener to the front plate influences the eigenfrequencies of the simplified gearbox. A general increase in their values is observed especially for the second class eigenvalues (front plate modes). This permit to avoid critical set of speed rotations and minimise the noise radiated by the casing of the gearbox. 


\section{CONCLUSION}

In this paper, the dynamic substructuring method is presented. Its implementation is made on a simplified gearbox. The driving and driven shafts connected to each other with a gear pair forms one substructure, while the casing or the front plate forms another substructure. The stiffener connected to the front plate forms a slave substructure in this case. The formulation for computing the vibratory response is based on a substructuring modal synthesis approach. Natural frequencies and modes of individual substructures subject to specific boundary conditions are used to compute the modal properties of assembled gearbox. Results obtained with dynamic substructuring method and finite element method are compared. A good agreement is observed. The originality of the dynamic substructuring method is to be able to consider one or several positions of the slave substructures without having to redo all the computational stages. This technique will be used in optimisation procedure of vibratory behaviour of complex mechanical structures.

\section{REFERENCES}

[1] Craig Jr, R. R. (1987). A review of time-domain and frequency-domain component mode synthesis methods, International Journal of Analytical and Experimental Modal Analysis, Vol. 2, No. 2, 59-72

[2] Cros, M. (1998). Parallel modal synthesis methods in structural dynamics, Contemporary Mathematics, Vol. 218, 492-499

[3] Hou, S. N. (1969). Review of modal synthesis technique and a new approach, Shock and Vibration Bulletin, Vol. 40, 25-39

[4] Craig Jr, R. R.; Bampton, M. C. C.; Chang, C. (1977). On the use of attachment modes in substructure coupling for dynamic analysis, Proceedings of the 18th Structures, Structural Dynamics and Materials Conference, San Diego, CA, 89-99

[5] Hurty, W. C. (1965). Dynamic analysis of structural systems using component modes, AIAA Journal, Vol. 3, 678-685

[6] Zakrajsek, J.; Oswald, B.; Townsend, P.; Coy, J. (1990). Gear noise vibration and diagnostic studied, NASA Lewis Research Centre, TM-102435

[7] Seybert, A. F.; Oswald, F. B. (1991). Acoustical analysis of gear housing vibration, NASA Lewis Research Centre, TM-103691

[8] Ozguven, H. N.; Houser, D. R. (1988). Mathematical-models used in gear dynamics, Journal of Sound and Vibration, Vol. 121, 383-660

[9] Todd, R.; Rajendra, S. (1996). Mobility analysis of structure-borne noise power flow through bearings in gearbox-like structures, Noise Control Engineering Journal, Vol. 44, No. 2, 69-78

[10] Adline, B. (1995). Modélisation dynamique globale des boites de vitesse automobile, Thèse de Doctorat, INSA. Lyon

[11] Rigaud, E.; Sabot, J.; Perret-Liaudet, J. (2000). Approche globale pour l'analyse de la réponse vibratoire d'une transmission par engrenages, Revue Européenne des Eléments Finis, Vol. 9, 315-330

[12] Lin, J.; Parker, R. G. (2002). Mesh stiffness variation instabilities in two-stage gear systems, Journal of Vibration and Acoustics, Vol. 124, 68-76

[13] Rigaud, E.; Barday, D. (1999). Modelling and analysis of wheel body deformation and interactions between adjacent loaded teeth, Proceeding of the 4th World Congress on Gearing and Power Transmission, Paris, Vol. 3, 1961-1972

[14] Batoz, J. L.; Dhatt, G. (1990). Modélisation des Structures par Eléments finis Poutres et Plaques, Edition Hermès, Paris

[15] Haddar, M. (1998). Réponse dynamique d'une boite de vitesse, Revue Internationale d'Ingénierie des Systèmes de Production Mécanique, Vol. 1, 29-34 